\title{
Distribution of 4-Nonylphenol Isomers in Surface Water of the Haihe River, People's Republic of China
}

\author{
X. Jin, ${ }^{1}$ G. Huang, ${ }^{1}$ G. Jiang, ${ }^{2}$ Q. Zhou, ${ }^{2}$ J. Liu ${ }^{2}$ \\ 1 College of Environmental Science and Engineering, Nankai University, Tianjin \\ 300071, People's Republic of China \\ 2 Key Laboratory of Environmental Chemistry and Ecotoxicology, Research Center \\ for Eco-Environmental Sciences, Chinese Academy of Sciences, Post Office Box \\ 2871, Beijing 100085, People's Republic of China
}

Received: 12 May 2004/Accepted: 8 September 2004

In the past decade, as one of the most important endocrine disrupters, 4-nonylphenols (NPs) have become a concerned topic in scientific literatures (Ferguson et al. 2001, and Isobe et al. 2001). NPs with branched nine-carbon alkyl chains attached to phenolic ring are considered as the products prepared by a Friedel-Crafts acid catalyzed reaction of phenol with nonene. NPs primarily use as intermediates in the manufacture of nonylphenol polyethoxylates (NPEOs), a major group of nonionic surfactants. NPs as metabolic products are also generated from the degradation of NPEOs in the environment. Approximately 400,000 tons annually produce in the world, NPEOs are largely manufactured and have wide industrial, agricultural and domestic applications (Ahel et al. 1996).

As NPs' obvious estrogenic effects on reproductive and development of animals (Jobling et al. 1996), it is important to understand their environmental distribution and fate. However, information on the estrogenic potential and distribution of individual isomeric NP is quite limited. Theoretically, different isomers of NPs should have different estrogenic activities and toxicities, but it is unproved because the individual pure isomer standards of NPs are not currently available (Thiele et al. 1997). It was reported that highly branched nonylphenols are more difficult to degrade in the environment (Pedersen et al. 1999 and Lalah et al. 2003a). Studies reporting environmental levels of NP isomers are scarcely any, even in heavily urbanized and industrialized areas.

Technical NPs consisting of $8,11,12,14,16$ or 18 isomers were characterized and reported in several previous papers (Thiele et al. 1997, Lalah et al. 2003b, Gundersen 2001, Espejo et al. 2002, Heemken et al. 2001 and Bhatt et al. 1992). Wheeler et al. (1997) separated and identified 22 isomers of NPs by HRGC-MS and GC-FTIR techniques. However, some individual peaks contained several isomers and did not represent pure isomers.

In this study, eighteen isomers of NPs were obtained after derivatization using trifluoroacetic anhydride. To investigate the contribution of the individual isomer to the environment, these isomers were divided into eleven compositions according to the results of separation and identification carried out by gas chromatograph-mass spectrometric with selected ion monitoring (GC-MS-SIM). 


\section{MATERIALS AND METHODS}

An Agilent 6890 gas chromatograph coupled with 5973 mass spectrometric detection was used to analyze NPs. GC separation was performed on a fused-silica capillary column [HP-5 MS (Hewlett-Packard); $30 \mathrm{~m} \times 0.25 \mathrm{~mm}$ i.d.; and film thickness $0.25 \mu \mathrm{m}]$. Helium, used as carrier gas, was controlled at a flow rate of $1.0 \mathrm{~mL} \cdot \mathrm{min}^{-1}$ in a constant flow mode. The oven temperature program was set as follows: the initial temperature was held at $80^{\circ} \mathrm{C}$ for $1 \mathrm{~min}$, then raised to $220^{\circ} \mathrm{C}$ at $10^{\circ} \mathrm{Cmin}^{-1}$, to $250^{\circ} \mathrm{C}$ at $20^{\circ} \mathrm{Cmin}^{-1}$, a post-analysis baking at $300^{\circ} \mathrm{C}$ for $3 \mathrm{~min}$. As to the MS parameters, electron impact ionization was set at $70 \mathrm{eV}$. The temperatures of interface, source and quadrupole were $280^{\circ} \mathrm{C}, 230^{\circ} \mathrm{C}$ and $150^{\circ} \mathrm{C}$, respectively. The injection was operated in pulsed splitless mode at a temperature of $250^{\circ} \mathrm{C}$. For the purposes of quantification, all samples were analyzed in SIM mode. The characteristic ions $m / z 203,217,231,245,259$ were selected as quantitative ions. The responses were calculated by using RTE integrator.

A technical mixture of 4-nonylphenols ( $>98 \%$ purity) was obtained from Tokyo Kasei Kogyo Co. LTD, Japan. Stock solution containing $44.6 \mathrm{mg} \cdot \mathrm{L}^{-1} \mathrm{NP}$ was prepared in methanol. Trifluoroacetic anhydride (TFAA) was obtained from Koch-Light Laboratories LTD, England. Pesticide grade Dichloromethane and n-hexane were purchased from FisherScientific. Acetone was of analytical reagent and redistilled before use. De-ionized water was double redistilled by quartz distiller. Analytical grade of 1,2,4-tribromobenzene (TBB), sodium chloride $(\mathrm{NaCl})$, hydrochloric acid $(\mathrm{HCl})$ and anhydrous sodium sulfate were all purchased from Beijing Chemicals Corporation.

As an important river in Tianjin city, Haihe's pollution has been of concern for a long period. It originates from the infalls of Beiyun, Nanyun and Ziya rivers, flows through downtown of Tianjin and Tanggu, and finally enters into Bohai Sea. In this study, fourteen water samples (R1-R14) were collected in the sampling sites in the Haihe River on April 8 and 9, 2003. Wherein, R1 was located at the estuary of the Haihe River, R2 belonged to urban of Tanggu, R3 to R7 were located at the area between Tianjin and Tanggu city, R8 to R11 existed in urban of Tianjin, R12, R13 and R14 were situated in three source rivers, respectively. Each sample was collected in $4 \mathrm{~L}$ of preconditioned amber glass bottles, filtered through $0.45 \mu \mathrm{m}$ cellulose acetate membrane filters, and extracted with dichloromethane as soon as possible.

After the addition of $15 \mathrm{~g}$ of $\mathrm{NaCl}$, the $\mathrm{pH}$ of water sample $(300 \mathrm{~mL})$ was adjusted to less than 2 by several drops of $50 \% \mathrm{HCl}(\mathrm{v} / \mathrm{v})$. Extraction was performed with $3 \times 20 \mathrm{~mL}$ of dichloromethane, shaken violently for three minutes each time. The combined organic phase was dehydrated with anhydrous sodium sulfate, concentrated by rotary evaporation to a volume of about $1 \mathrm{~mL}$, then transferred to a reaction vial and gently evaporated to dryness under a stream of high pure 


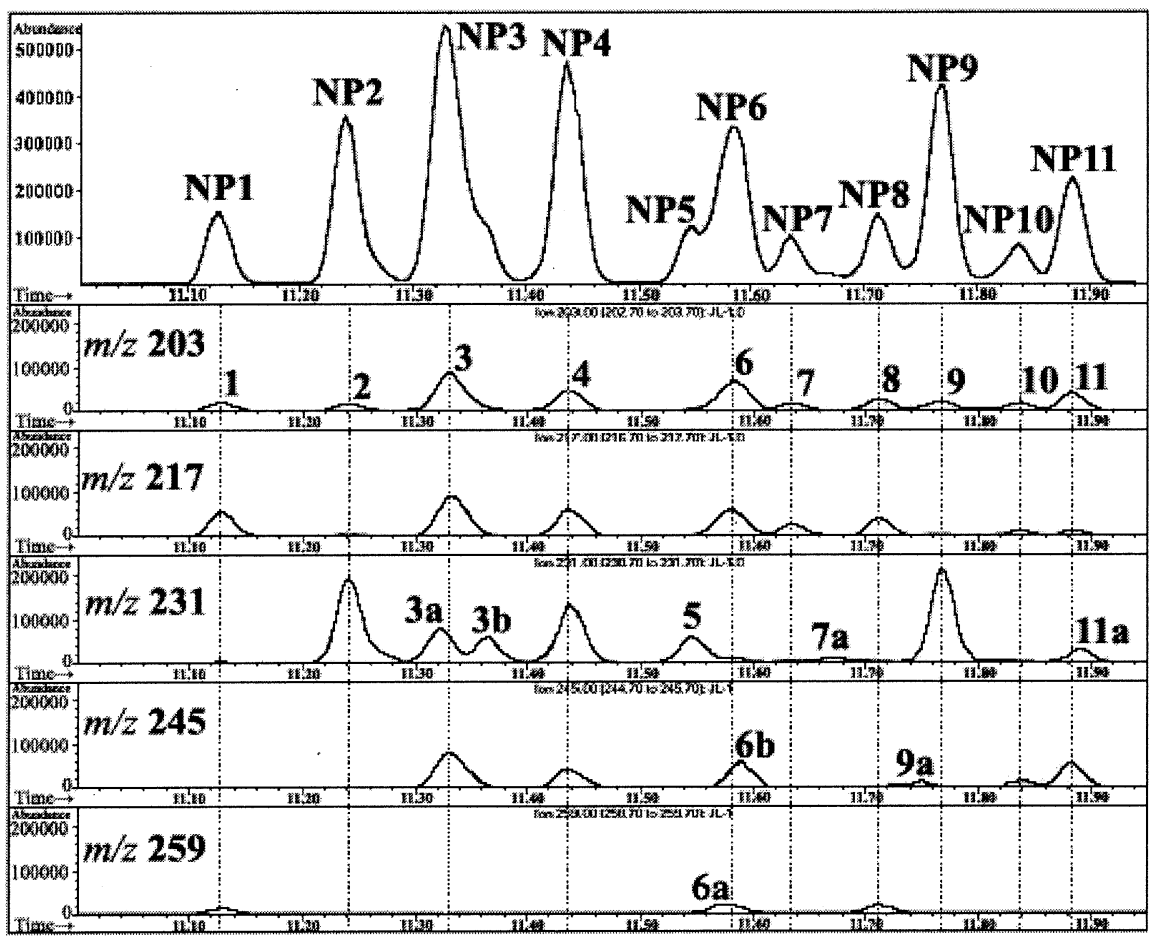

Figure 1. TIC and EIC of the NP-TFAO isomers with GC-MS.

nitrogen. Derivatization was rapidly performed with $100 \mu \mathrm{L}$ of TFAA (Varelis et al. 2000 , Rinken 2001). The reaction vial was sealed and kept in $30{ }^{\circ} \mathrm{C}$ water bath for $10 \mathrm{~min}$, then sonicated for $10 \mathrm{~min}$ in $30^{\circ} \mathrm{C}$ water bath. The products were evaporated to dryness under a stream of nitrogen. The residues were dissolved in $100 \mu \mathrm{L}$ of $\mathrm{n}$-hexane containing $0.4 \mathrm{mg} \cdot \mathrm{L}^{-1}$ of $\mathrm{TBB}$ as internal standard of quantitative analysis. An aliquot of $1 \mu \mathrm{L}$ of the derived resultants was injected for GC-MS analysis.

\section{RESULTS AND DISCUSSION}

The total ion chromatogram (TIC) including eleven peaks of the derivatized NPs (NP-TFAO) was obtained by GC-MS with scan mode and shown in Figure 1. For the further identification, the TIC was subjected to extract ion fragment analysis. Based on extract ion chromatograms (EIC) and mass spectra, the characteristic ions of $\mathrm{m} / \mathrm{z} 203,217,231,245$ and 259 were selected as quantitative ions of NP-TFAO isomers using GC-MS-SIM. They were well consistent with the characteristic ions of $\mathrm{m} / \mathrm{z} 107,121,135,149$ and 163 for NP isomers. Because the ion of $m / z 149$, one of the characteristic ions of NP isomers, could also come from the fragmentation of phthalate esters widely distributing in the environment, it was possible that phthalate esters may interfere with the analysis of NPs. Fortunately, the interference did not occur once the NP was derivatized by TFAA. It was 
demonstrated that derivatization step could greatly improve the separation of NPs.

Comparing the EIC of NPs with that of NP-TFAOs, it was found that NP-TFAOs were better separated than NPs. It was likely that the structures and the cleavage patterns were not changed after derivatization by TFAA because the numbers of isomers remained the same in the EIC of NPs and NP-TFAOs. But the separation of NP-TFAOs differed from the NPs owing to the polarity of NP-TFAOs varied after derivatization. The retention time of NP-TFAOs was shorter than that of NPs, and the elution order of individual NP-TFAO isomer did not correspond to the NP isomers one by one.

The characteristic ions of NP-TFAO were selected and applied to quantify the levels of the NP isomers. However, eleven peaks of NP-TFAO in TIC were attributed to the different isomers of the NP trifluoroacetates, some individual peaks did not represent single isomer but contained several isomers. According to the comparison of NP-TFAO isomers' TIC with their EIC shown in Figure 1, peak NP3 in TIC was a mixture of peak 3, 3a and 3b in EIC. Peak NP6 in TIC contained peak $6,6 \mathrm{a}$ and $6 \mathrm{~b}$ in EIC. Peak NP9 and NP11 in TIC contained peak 9, 9a and 11, 11a in EIC, respectively. Peak 7a in EIC was an independent compound, but it was not conspicuous in TIC because of its small quantity. This result indicated some NP isomers were similar in the structure, and some occupied a slight proportion.

The proportion of each individual NP peak area to the sum area of all peaks was different in standards or samples. The proportion of the same individual NP composition possessed distinguished between standards and samples. In the standards, the proportion of each NP composition almost maintained unchanged with RSD less than $8.3 \%$ at different levels. The proportion of NP1 to NP11 in the standards was $0.05,0.12,0.23,0.15,0.03,0.13,0.03,0.05,0.12,0.03,0.07$, and RSD was $6.1,5.1,1.5,2.2,8.3,3.5,5.0,4.6,2.4,5.0,3.9$, respectively. The proportions were more than 0.1 in NP2, NP3, NP4, NP6 and NP9 with a dominated order of NP3 $>$ NP4 $>$ NP6 $>$ NP2 $>$ NP9 at various levels. However, the proportion of each NP composition varied significantly in the surface water samples with RSD ranging from $9.1 \%$ to $42.8 \%$, and the average proportion of each NP composition in the samples were great different from standards. The proportion of NP1 to NP11 in the standards was $0.06,0.12,0.21,0.13,0.02,0.17$, $0.05,0.08,0.08,0.02,0.06$ and RSD was $15.6,13.6,10.7,9.1,22.6,14.2,20.6$, $16.6,26.0,42.8,10.7$, respectively. In the samples, the proportions were larger than 0.1 in NP2, NP3, NP4 and NP6 with an order of NP3 $>$ NP6 $>$ NP4 $>$ NP2 at different sampling sites. This result indicated the proportion of each NP composition in environmental samples was not stable as that in the standard. Therefore, quantification using the sum of NP was inappropriate; it covered up the beneficial information on the compositions of NP.

According to the above factors, quantification of NP isomers was only performed with the separated eleven peak compositions including eighteen isomers in TIC of NP-TFAO shown in Figure 1. All characteristic signals of the compositions are presented in all standards and water samples. The peak areas of eleven selected 
Table 1. The parameters of the proposed procedure and the levels of various nonylphenol compositions in all samples using GC-MS-SIM.

\begin{tabular}{|c|c|c|c|c|c|c|c|}
\hline Peak & Linear equation & $\mathrm{R}^{2}$ & $\begin{array}{c}\text { Recoveries } \\
(\%)\end{array}$ & $\begin{array}{c}\text { RSD } \\
(\%)\end{array}$ & \multicolumn{3}{|c|}{ Levels $\left(\mathrm{ng} \cdot \mathrm{L}^{-1}\right)$} \\
\hline NP1 & $\mathrm{A}_{1} / \mathrm{A}_{\mathrm{TBB}}=0.0195 \mathrm{C}_{\mathrm{NP} 1}-0.0842$ & 0.9976 & 124 & 4 & 7.1 & 11.4 & 39.1 \\
\hline & $\mathrm{A}_{2} / \mathrm{A}_{\mathrm{TBB}}=0.0179 \mathrm{C}_{\mathrm{NP} 2}-0.1662$ & 0.9980 & & 5 & 12.8 & 24.4 & 53.4 \\
\hline P3 & $\mathrm{A}_{3} / \mathrm{A}_{\mathrm{TBB}}=0.0181 \mathrm{C}_{\mathrm{NP} 3}-0.2729$ & 0.9976 & 111 & 12 & 20.5 & 40.6 & 151.2 \\
\hline $\mathrm{P} 4$ & $\mathrm{~A}_{4} / \mathrm{A}_{\mathrm{TBB}}=0.0182 \mathrm{C}_{\mathrm{NP} 4}-0.2032$ & 0.9983 & & 9 & 16.3 & 26.2 & 89.6 \\
\hline NP5 & $\mathrm{A}_{5} / \mathrm{A}_{\mathrm{TBB}}=0.0$ & & 129 & 14 & 3.6 & 5.9 & 22.7 \\
\hline P6 & $\mathrm{A}_{6} / \mathrm{A}_{\mathrm{TBB}}=0.017$ & & 108 & 12 & 15.9 & 30.5 & 73.4 \\
\hline IP7 & $\mathrm{A}_{7} / \mathrm{A}_{\mathrm{TBB}}=0.0174 \mathrm{C}_{\mathrm{NP} 7}-0.0325$ & 0.9973 & 123 & 16 & 3.9 & 7.8 & 16.1 \\
\hline NP8 & $\mathrm{A}_{8} / \mathrm{A}_{\mathrm{TBB}}=0.0184 \mathrm{C}_{\mathrm{NP} 8}-0.0566$ & 0.9984 & 111 & 11 & 6.1 & 13.6 & 23.8 \\
\hline NP9 & $\mathrm{A}_{9} / \mathrm{A}_{\mathrm{TBB}}=0.0182 \mathrm{C}_{\mathrm{NP} 9}-0.1547$ & 0.9984 & 98 & 11 & 10.9 & 18.5 & 46.0 \\
\hline NP10 & $\mathrm{A}_{10} / \mathrm{A}_{\mathrm{TBB}}=0.0189 \mathrm{C}_{\mathrm{NP} 10}-0.0354$ & 0.9985 & 99 & 9 & 2.6 & 4.2 & 7.7 \\
\hline JP11 & $\mathrm{A}_{11} / \mathrm{A}_{\mathrm{TBB}}=0.0190 \mathrm{C}_{\mathrm{NP} 11}-0.0985$ & 0.9982 & 97 & 9 & 6.8 & 12.4 & 31.1 \\
\hline
\end{tabular}

peaks should be integrated individually, and then compared with the peak area of TBB. Calibration curves of the NP compositions were established with a series of standard solutions described in the proposed procedure. The linear regression equations and the regression coefficients were obtained and shown in Table 1, which indicated that good linearity was obtained within the corresponding concentration range. To validate the recovery and reproducibility of the method, sample R1 was spiked with an appropriate level of NPs standard and the results were shown in Table 1. The satisfied recoveries (87-133\%) and RSDs (4-16\%, n=4) were obtained which indicated the proposed procedure was reliable and suitable for water sample analysis.

The proposed method was applied to determine the levels of NP compositions in surface water samples and results obtained were shown in Figure 2. The levels of NPs were summed up with the levels of each individual composition. The contributive proportions of individual NP composition level to the level of NPs shown in Table 2 were different. The contributive proportion of NP3 dominated in all samples, and the order of major contributor (more than 0.1 ) was accordant to NP3 $>$ NP6 $>$ NP4 $>$ NP2 in all samples. The results in Table 2 demonstrated that the contributive proportion of individual NP composition was not equal in different sampling sites. Figure 2 also indicated the level of the same individual NP composition was dissimilar in each samples. Although each NP composition levels in sample R7 shown in Figure 2 were the lowest, the contributions of NP compositions in this sample (listed in Table 2) were not the lowest one. Apart from NP7 in Figure 2, the levels of NP compositions in sample R1 were higher than those in other samples.

The levels of various NP compositions were varied with the sampling sites. The tendency of the NP7 composition level in sampling sites was similar to that of NP8. The levels of the other compositions were shown in Figure 2, respectively. 

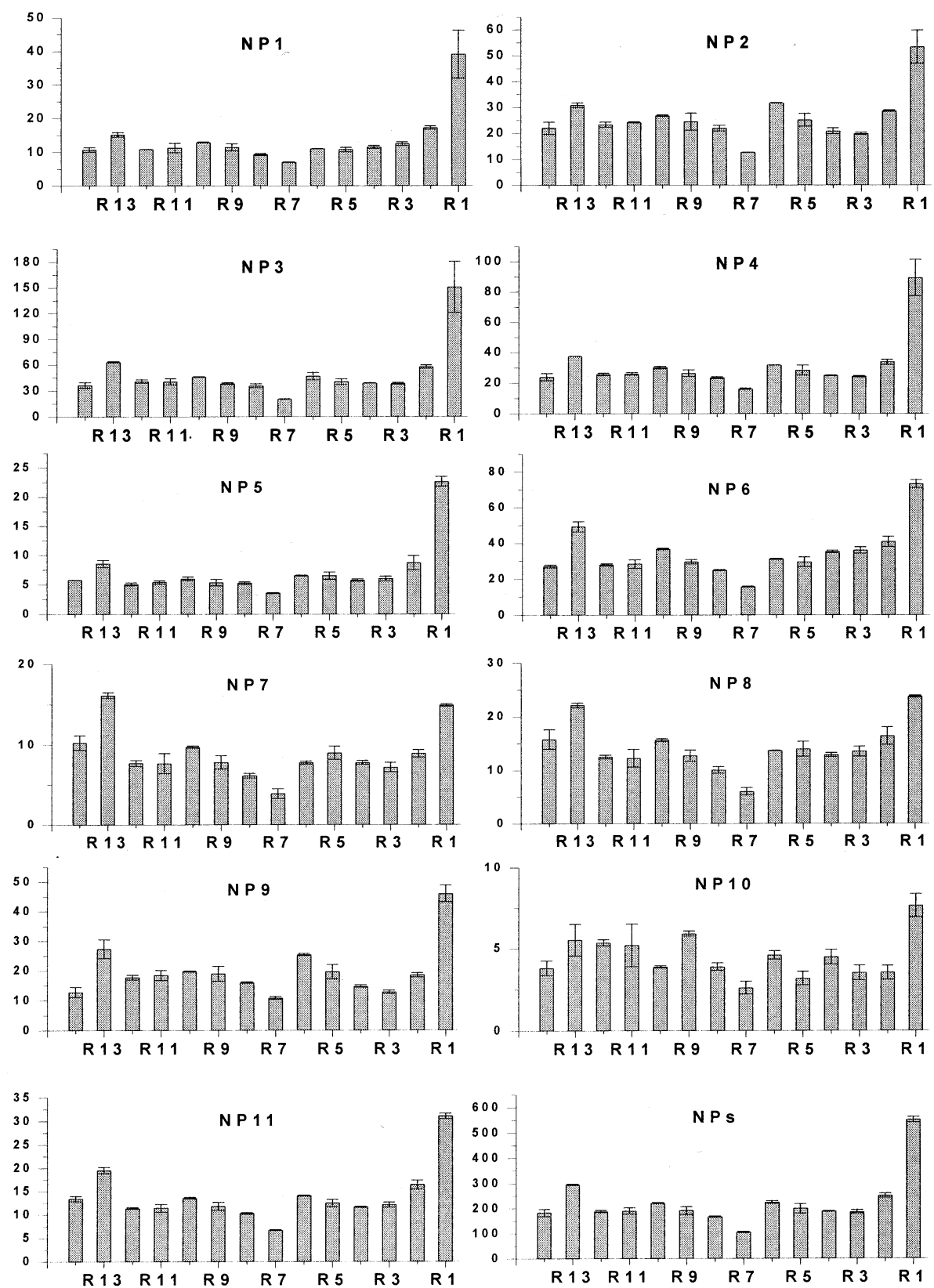

Figure 2. Levels of various nonylphenol compositions in surface water samples. The vertical axis represents the level of nonylphenol compositions, the unit is $\mathrm{ng} \cdot \mathrm{L}^{-1}$. The horizontal axis represents the sampling sites. 
Table 2. Proportions of various nonylphenol compositions level relative to the total levels of all compositions in different samples.

\begin{tabular}{lcccccccccccccc}
\hline & R14 & R13 & R12 & R11 & R10 & R9 & R8 & R7 & R6 & R5 & R4 & R3 & R2 & R1 \\
\hline NP1 & 0.06 & 0.05 & 0.06 & 0.06 & 0.06 & 0.06 & 0.06 & 0.07 & 0.05 & 0.05 & 0.06 & 0.07 & 0.07 & 0.07 \\
NP2 & 0.12 & 0.10 & 0.12 & 0.13 & 0.12 & 0.13 & 0.13 & 0.12 & 0.14 & 0.13 & 0.11 & 0.11 & 0.11 & 0.10 \\
NP3 & 0.20 & 0.21 & 0.22 & 0.21 & 0.21 & 0.20 & 0.21 & 0.19 & 0.21 & 0.20 & 0.21 & 0.21 & 0.23 & 0.27 \\
NP4 & 0.13 & 0.13 & 0.14 & 0.14 & 0.14 & 0.14 & 0.14 & 0.15 & 0.14 & 0.14 & 0.13 & 0.13 & 0.14 & 0.16 \\
NP5 & 0.03 & 0.03 & 0.03 & 0.03 & 0.03 & 0.03 & 0.03 & 0.03 & 0.03 & 0.03 & 0.03 & 0.03 & 0.04 & 0.04 \\
NP6 & 0.15 & 0.17 & 0.15 & 0.15 & 0.17 & 0.15 & 0.15 & 0.15 & 0.14 & 0.15 & 0.19 & 0.19 & 0.16 & 0.13 \\
NP7 & 0.06 & 0.06 & 0.04 & 0.04 & 0.04 & 0.04 & 0.04 & 0.04 & 0.04 & 0.05 & 0.04 & 0.04 & 0.04 & 0.03 \\
NP8 & 0.09 & 0.08 & 0.07 & 0.06 & 0.07 & 0.07 & 0.06 & 0.06 & 0.06 & 0.07 & 0.07 & 0.07 & 0.07 & 0.04 \\
NP9 & 0.07 & 0.09 & 0.09 & 0.10 & 0.09 & 0.10 & 0.10 & 0.10 & 0.11 & 0.10 & 0.08 & 0.07 & 0.07 & 0.08 \\
NP10 & 0.02 & 0.02 & 0.03 & 0.03 & 0.02 & 0.03 & 0.02 & 0.03 & 0.02 & 0.02 & 0.02 & 0.02 & 0.01 & 0.01 \\
NP11 & 0.07 & 0.07 & 0.06 & 0.06 & 0.06 & 0.06 & 0.06 & 0.06 & 0.06 & 0.06 & 0.06 & 0.07 & 0.07 & 0.06 \\
\hline
\end{tabular}

The different sampling sites resulted in the above complex conclusion. It was likely that the high contributive compositions were persistent, difficult to degradation or derived abundantly from parent compounds (NPEOs) in environmental sample. On the contrary, the low one could be easy to degrade and be lack of source. In sample R1, the levels of various compositions appealed evidently in all samples. This phenomenon was related to the estuarine character of the Haihe River, which flows through industrial districts and several pollutant sources such as thermoelectric plant, a harbor located ahead of R1 and so on. A chemical plant and a sewage outlet nearby R1, the effluent may easily pollute the estuary of the Haihe River (R1) during the period of flood tide. The sampling site R7 was located between Tianjin and Tanggu, middle reach of the Haihe River. The contaminants from upstream of the Haihe River and urban of Tianjin were gradually self-purified or could be diluted by the enhanced hydraulic load of river. The sampling site R7 avoided the contamination from ambient and Tanggu industrial estate. Hence, the levels of various compositions in sample R7 were much less than the others. The distributions of various compositions levels were presented in Figure 2 and summarized in Table 1.

Acknowledgments. We are grateful to the National Natural Science Foundation of China (20137010) and the High Technology Research and Development (863) Programme of China (2003AA640610) for supporting this work.

\section{REFERENCES}

Ahel M, Schaffner C, Giger W (1996) Behaviour of alkylphenol polyethoxylates surfactants in the aquatic environment - III Occurrence and elimination of their persistent metabolites during infiltration of river water to groundwater transformation in rivers. Wat Res 30:37-46

Bhatt BD, Prasad JV, Kalpana G, Ali S (1992) Separation and Characterization of 
isomers of p-nonylphenols by capillary GC/GC-MS/GC-FTIR techniques. J Chromatogr Sci 30:203-210

Espejo R, Valter K, Simona M, Janin Y, Arrizabalaga P (2002) Determination of nineteen 4-alkylphenol endocrine disrupters in Geneva municipal sewage wastewater. J Chromatogr A 976:335-343

Ferguson PL, Charles RI, Brownawell BJ (2001) Analysis of nonylphenol and nonylphenol ethoxylates in environmental samples by mixed-mode high-performance liquid chromatography-electrospray mass spectrometry. J Chromatogr A 938:79-91

Gundersen JL (2001) Separation of isomers of nonylphenol and select nonylphenol polyethoxylates by high-performance liquid chromatography on a graphitic carbon column. J Chromatogr A 914:161-166

Heemken OP, Reincke H, Satchel B, Theobald N (2001) The occurrence of xenoestrogens in the Elbe river and the North Sea. Chemosphere 45:245-259

Isobe T, Nishiyama H, Nakashima A, Takada H (2001) Distribution and Behavior of Nonylphenol, Octylphenol, and Nonylphenol Monoethoxylate inTokyo Metropolitan Area: Their Association with Aquatic Particles and Sedimentary Distributions. Environ Sci Technol 35:1041-1049

Jobling S, Sheahan D, Osborne JA, Matthiessen P, Sumpter JP (1996) Inhibition of testicular growth in rainbow trout (Oncorhynchus mykiss) exposed to estrogenic alkylphenolic chemicals. Environ Toxicol Chem 15:194-202

Lalah JO, Schramm KW, Severin GF, Lenoir D, Henkelmann B, Behechti A, Guenther K, Kettrup A (2003a) In vivo metabolism and organ distribution of a branched 14C-nonylphenol isomer in pond snails (Lymnaea stagnalis L.). Aquat Toxicol 62:305-319

Lalah JO, Schramm KW, Henkelmann B, Lenoir D, Behechti A, Gunther K, Kettrup A (2003b) The dissipation, distribution and fate of a branched ${ }^{14} \mathrm{C}$-nonylphenol isomer in lake water/sediment systems. Environ Pollut 122:195-203

Pedersen SN, Christiansen LB, Pedersen KL, Korsgaard B, Bjerregaard P (1999) In vivo estrogenic activity of branched and linear alkylphenols in rainbow trout Oncorhynchus Mykiss. Sci Total Environ 233:89-96

Rinken MJ (2001) GC-MS determination of bisphenol A in water at nanogram per liter concentrations. Intern J Environ Anal Chem 82:77-85

Thiele B, Günther K, Schwuger MJ (1997) Alkylphenol Ethoxylates: Trace Analysis and Environmental Behavior. Chem Rev 97:3247-3272

Varelis P, Balafas D (2000) Preparation of 4,4'-(1-[ $\left.{ }^{2} \mathrm{H}_{6}\right]$ methylethylidene) bis-[2, $\left.3,5,6-{ }^{2} \mathrm{H}_{4}\right]$ phenol and its application to the measurement of bisphenol $\mathrm{A}$ in beverages by stable isotope dilution mass spectrometry. J Chromatogr A 883: $163-170$

Wheeler TF, Hein JR, Latorre MR, Janes AB (1997) Mass spectral charaterization of p-nonylphenol isomers using high-resolution capillary GC-MS. J Chromatogr Sci 35:19-30 